\title{
Condiciones acústicas de las aulas universitarias en una Universidad pública en Bogotá
}

\section{Classroom acoustics conditions at public University in Bogotá}

\section{Lady Catherine Cantor Cutiva}

Fonoaudióloga.

Universidad Nacional de Colombia. Bogotá (Colombia)

\section{Alba Idaly Muñoz Sánchez}

Doctora en Enfermería

Universidad Nacional de Colombia. Bogotá (Colombia)

Correspondencia:

Lady Catherine Cantor Cutiva

Fonoaudióloga. Magíster en Salud y Seguridad en el Trabajo

Universidad Nacional de Colombia

Dirección: Calle 22A \# 1-50 Barrio Las Aguas. Bogota, Colombia

Tfno. Móvil: 3005507274

e-mail: ladyccantor@gmail.com

Resumen

Introducción: En el sector de la docencia una situación frecuente son las precarias condiciones acústicas de las aulas.

Objetivo: Caracterizar las condiciones acústicas de algunas aulas de una universidad pública en Bogotá.

Material y Métodos: Durante el I semestre del 2009 se realizó un estudio descriptivo transversal, se evaluaron 25 salones de una universidad pública de Bogotá, los cuales fueron seleccionados de forma aleatoria según los reportes de docentes previamente entrevistados.

Resultados: De los 25 salones medidos en la facultad 1 el 63,63\% de los salones medidos presentó muy mala acústica según el índice de Acústica Total, mientras que en la Facultad 2 el 40\% de los salones tuvo mala acústica, y en la Facultad 3 el 33,3\% presentó acústica mala.

Conclusiones: La ausencia de estudios en los que se reporten mediciones objetivas de tiempo de reverberación (TR) e inteligibilidad evidencia la necesidad de realizar disertaciones de este tipo buscando generar herramientas que permitan mejorar las condiciones de salud, trabajo y vida de este grupo ocupacional.

(Med Segur Trab (Internet) 2009; 55 (216): 26-34)

Palabras claves: acústica, proceso de trabajo, ergonomía 


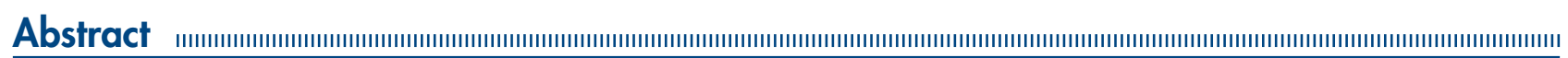

Introduction: Teachers complain about bad acoustic conditions in their classroom frequently.

Aim: to describe some classroom acoustic conditions at Colombian Estate University in Bogotá city.

Material and Methods: a transversal descriptive study was development in the first semester of 2009, 25 classrooms was measured at Colombian University in Bogota City, this classroom was selected randomized way according preview teachers evaluated reporting.

Results: about 25 measured classroom at Faculty 1 63.63\% of classroom had acoustic bad according Total Acoustic Index, while at Faculty 2 40\% of classroom has bad acoustic, and Faculty $333.3 \%$ had bad acoustic.

Conclusions: studies to report reverberation time (TR) and intelligibility objectives measurements are a few, this exposed it is necessary to do dissertations to investigate and to contribute tools according to determinate better health, job and life conditions in this occupational group.

(Med Segur Trab (Internet) 2009; 55 (216): 26-34)

Key words: acoustic, work process, ergonomics 


\section{INTRODUCCIÓN}

La promoción de la salud en los lugares de trabajo (PSLT) dirige sus esfuerzos a la generación de herramientas que incluyan los diferentes actores de la sociedad (comunidad, empleadores, empleados) para mejorar la salud de las personas en los lugares de trabajo (Casas \& Klijn, 2006). Debido a la frecuente exposición de los docentes a diferentes condiciones de trabajo que los afectan física, psíquica y socialmente, influyendo en su salud, bienestar y calidad de vida; se deben proponer herramientas que favorezcan sus condiciones de salud-trabajo-vida a partir del análisis de los actuales ambientes laborales y la aproximación a propuestas reales que optimicen el ambiente de trabajo actual

El objetivo del presente estudio es caracterizar las condiciones acústicas de algunos salones de tres facultades de una universidad pública en Bogotá, Colombia.

Durante el I semestre del 2009 se realizaron mediciones acústicas de los salones en que algunos docentes entrevistados previamente habían reportado realizar la mayoría de las actividades académicas, estas mediciones ambientales fueron realizadas con el apoyo de un ingeniero de sonido y de un ingeniero ocupacional; y consistían en tomar tres puntos en cada salón en los cuales se media el tiempo de reverberación luego de producido un sonido de impacto.

En la presente investigación el sonido fue producido por un globo, el cual era explotado desde un punto fijo del aula, cada vez que se realizaba una medición, para el proceso se determinó que todos los globos fueran de tamaño similar, y fueran inflados bajo las mismas condiciones, buscando homogeneizar las mediciones realizadas. La información recolectada en las valoraciones individuales a los docentes, y en las mediciones ambientales en los salones fue analizada con el software SPSS 11.5.

Dentro de los principales resultados se encontró que de los 25 salones medidos en la facultad 1 el 63,63\% de los salones presentó muy mala acústica según el índice de Acústica Total, mientras que en la Facultad 2 el 40\% de los salones tuvo mala acústica, y en la Facultad 3 el 33,3\% presentó acústica mala.

Lo anterior permite concluir que el docente universitario está sumergido en una serie de dinámicas interaccionales y organizacionales que determinan las exigencias y el desarrollo de la tarea; tales como la carga académica, el número de estudiantes por clase, las condiciones físicas de los espacios en los que se realizan las actividades académicas, además de otras situaciones generan que las demandas a las que se debe enfrentar el profesor se incrementen, influyendo directamente en sus condiciones de salud-trabajo-vida.

\section{MATERIAL Y MÉTODOS}

Durante el I semestre del 2009 se realizó un estudio descriptivo transversal cuyo objetivo era describir las condiciones acústicas de algunos de los salones de tres facultades en los cuales se realizan actividades académicas en una universidad pública en Bogotá.

Los 25 salones evaluados se distribuyeron en las tres facultades de la siguiente forma: facultad $1=11$ salones, facultad $2=5$ salones, facultad $3=9$ salones. Este tamaño de población suministra suficiente información para tener una base del comportamiento acústico de las aulas de las tres facultades. Aunque, las conclusiones obtenidas bajo esta muestra, no se pueden generalizar para toda la población, se pueden considerar como potenciales hipótesis para el desarrollo de otra investigación. Además alertan sobre las condiciones de trabajo de los docentes que desarrollan sus actividades en estos espacios.

Por otro lado, al realizar la revisión bibliográfica que enmarcó la investigación se obtuvieron diversos instrumentos que fueron adaptados para los propósitos del estudio, en el aspecto acústico, se adaptaron los documentos de Harris (1991) y las normas ISO 354 y UNE-EN-ISO 140-4; las mediciones fueron realizadas con el apoyo técnico de un ingeniero de sonido y un ingeniero ocupacional. 
En cuanto a los instrumentos, para las mediciones realizadas se utilizó un Micrófono de medición Behringer ECM8000, una tarjeta de sonido Digidesign audio Mbox 2, un stand de micrófono, y un computador portátil compaq presario. Para la determinación de los tiempos de reverberación y la inteligibilidad del habla se utilizaron los softwares protools 7.1 número de licencia 99001724600 y, plugin AURORA de descarga gratis para análisis acústico.

En cada aula se realizaron tres mediciones a diferentes distancias de la fuente sonora, antes de iniciar cada jornada de medición se corroboraba que los equipos estuvieran calibrados y el aula vacía; luego se ubicaba el micrófono en un punto central en el salón, se medía la distancia y se producía el estimulo sonoro de impacto (explosión de un globo), el procedimiento se repetía dos veces más a diferentes distancias tratando de abarcar la magnitud del salón. El ingeniero acústico determinaba el tiempo de reverberación y la inteligibilidad en cada punto. Para sacar el Tiempo de Reverberación promedio de cada aula, se sumaron los tres valores encontrados y se dividieron en 3 .

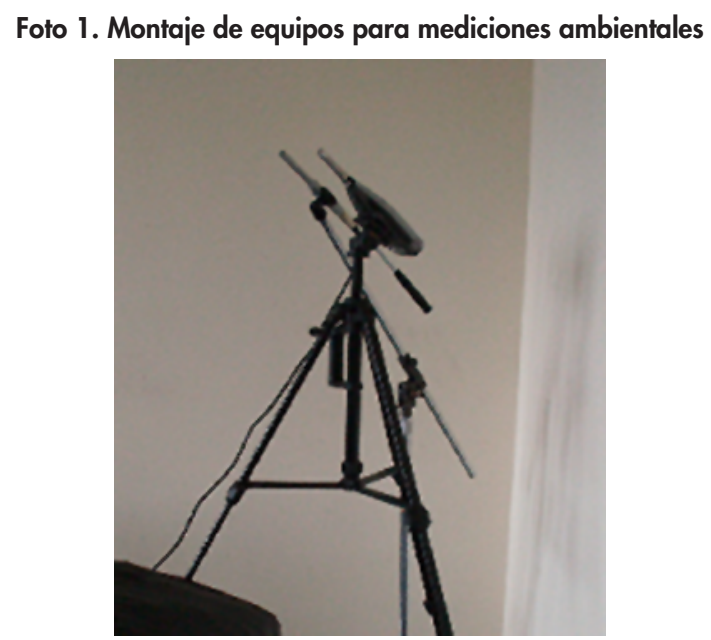

Se realizó una base de datos en Excel a partir de la información recolectada en la entrevista de los docentes y la muestra de voz, la caracterización y análisis descriptivo de las condiciones de trabajo de los docentes fue realizada con el software SPSS 11,5.

\section{RESULTADOS}

Con el objetivo de identificar y describir las condiciones acústicas de las aulas en las que algunos docentes reportaron dictar la mayoría de sus clases, se realizaron mediciones ambientales de tiempo de reverberación e inteligibilidad, encontrando los siguientes hallazgos:

Los tiempos de reverberación de todos los salones evaluados en las tres facultades fueron muy amplios según el volumen de los mismos, sin embargo, en la facultad 1 estos valores fueron mucho más amplios de lo sugerido por la Recomendación Biap (en ocasiones duplicaban el valor).

TABLA 1. Clasificación de tiempos de reverberación por facultades

\begin{tabular}{ccccc}
\hline FACULTAD & BUENO & REGULAR & TR MALO & TR MUY MALO \\
\hline $\mathbf{1}$ & 0 & 0 & 0 & 11 \\
$\mathbf{2}$ & 0 & 0 & 4 & 1 \\
\hline
\end{tabular}


El 100\% de los salones de la facultad 1 tienen el TR muy malo; el $80 \%$ de los salones de la facultad 2 tienen el TR malo y el 20\% muy malo; y el 66,6\% de los salones de la facultad 3 tienen el TR malo y el 33,3\% muy malo.

Respecto a la inteligibilidad de los salones evaluados, aunque es mala en toda la muestra, es nuevamente, en la facultad 1 en la que hay peor inteligibilidad, el 63,63\% de los salones tienen pobre inteligibilidad y el 36,36\% inteligibilidad regular. En la facultad 2 el $80 \%$ de los salones tienen inteligibilidad regular y el $20 \%$ pobre; y en la facultad 3 el $100 \%$ de los salones tienen inteligibilidad regular.

TABLA 2. Clasificación de Inteligibilidad

\begin{tabular}{cccc}
\hline FACULTAD & BUENA & REGULAR & POBRE \\
\hline $\mathbf{1}$ & 0 & 4 & 7 \\
$\mathbf{2}$ & 0 & 4 & 1 \\
$\mathbf{3}$ & 0 & 9 & 0 \\
\hline
\end{tabular}

Debido a que la acústica de un recinto está dada en función del Tiempo de Reverberación y la Inteligibilidad, se optó por determinar un índice de Acústica Total analizando estas dos variables en cada salón. Se encontraron los siguientes resultados:

TABLA 3. Acústica Total

\begin{tabular}{ccccc}
\hline FACULTAD & BUENA & REGULAR & MALA & MUY MALA \\
\hline $\mathbf{1}$ & 0 & 0 & 4 & 7 \\
$\mathbf{2}$ & 0 & 3 & 2 & 0 \\
\hline
\end{tabular}

En la facultad 1 el 36,36\% presentó mala acústica según el índice de Acústica Total, mientras que el 63,63\% de los salones medidos presentó muy mala acústica. En la Facultad 2 el 60\% de los salones tuvo acústica regular, mientras el $40 \%$ mala acústica. En la Facultad 3 el 66,6\% presentó regular acústica y el 33,3\% acústica mala.

Dentro de los salones evaluados ninguno obtuvo medidas de buena inteligibilidad, los salones estuvieron en un rango de regular a muy mala inteligibilidad.

\section{DISCUSIÓN}

Es importante resaltar que debido a la naturaleza de la presente investigación los resultados obtenidos no pueden generalizarse y sólo dan razón de los espacios evaluados; sin embargo, se resalta la importancia de realizar futuras investigaciones que determinen el impacto de las características acústicas en las condiciones de saludtrabajo-vida de los docentes.

Por otro lado, la Educación Nacional actualmente está influenciada por de las políticas de globalización y neoliberalismo que determinan la modernidad mundial, la universidad pública no es ajena a esta situación, y por ende las condiciones y los procesos de trabajo que en ella se desarrollan tampoco, por esta razón al describir algunas de las condiciones de trabajo de los docentes evaluados de la universidad participante es imperativo contextualizar el proceso de trabajo teniendo en cuenta los procesos de globalización y políticas neoliberales, que de forma directa afectan las políticas educativas actuales. 
La globalización también influye en las políticas educativas y sus procesos, entre otros, demandando a las instituciones de educación superior estrategias especificas para su funcionamiento, en este proceso la concepción de la educación se transforma de servicio a producto, lo que implica el deterioro de la misma debido al desgaste que se va presentando en las infraestructuras, las tecnologías y el talento humano.

De esta manera, cuando se deja de reconocer a la educación como un servicio y un bien de los ciudadanos, y se le cataloga como un producto "autofinanciable", el mantenimiento de las instituciones se puede ver condicionado por la oferta y la demanda de éste, y al caer en ese intercambio, se pierde de vista las condiciones internas en las que se realiza el proceso educativo.

Bajo esta perspectiva se vuelve determinante "la imagen del producto" más que "el producto" en sí, y es en este contexto en el que actualmente pueden estar incursionando los docentes de la universidad pública, enfrentándose a situaciones que les demandan mayor desgaste físico y mental con el objetivo de cubrir las demandas de la universidad.

Por otro lado, bajo las políticas del pensamiento neoliberal, la educación además de convertirse en un producto, se ve sujeta a las fuerzas del mercado, con oportunidad de inversión y ganancia; cuya producción debe ser eficiente, eficaz, rentable y de calidad.

Se sugiere que en este dilema la universidad inicia sus propuestas de reforma, e inicia el proceso de incremento de la cobertura y puede sacrificar las condiciones de trabajo de sus profesores, ya que se aumenta la cobertura, se cumplen las políticas de equidad social ofreciendo educación superior a los estratos menos favorecidos, pero todo lo anterior se realiza con el mismo personal docente.

No se puede desconocer que la universidad busca dar respuesta a las políticas nacionales, que actualmente refieren que el Estado tiene la responsabilidad de crear las condiciones necesarias para que todos los individuos puedan acceder de forma equitativa a la educación como una propuesta de equidad social, y simultáneamente exigen a las instituciones educativas ser rentables y de calidad (Miretti y Santiago, 2003). Sin embargo, en este afán se pueden orientar políticas y acciones que no prioricen las condiciones de trabajo adecuadas para sus docentes trabajadores, generando y/o aumentando factores de riesgo físicos y mentales que enfatizan el proceso de salud-enfermedad del profesor como trabajador; ya que, entre otras cosas, las cargas laborales se aumentan para garantizar el mantenimiento de la institución al incrementar la oferta educativa con la misma infraestructura y con el mismo talento humano.

Por otro lado, existen recomendaciones mundiales de modernidad y políticas educativas (Miretti y Santiago, 2003) que sugieren que la maquinaria educativa debe implementar mecanismos estructurales, tecnológicos, didácticos y humanísticos que garanticen el acceso masivo y equitativo a la educación, sin disminuir la calidad de la misma.

Este es el contexto organizacional y sociocultural que delimita el proceso de trabajo docente, y por ende las condiciones intralaborales que enmarcan la tarea docente en la universidad, tales como carga horaria, estudiantes por curso, condiciones físicas de los espacios en los que se realizan las clases, etc.

En el presente estudio se enfatiza en las condiciones acústicas, ya que varios autores las determinan como factores de riesgo para el desarrollo de patología vocal (Simões y Dias, 2006; Ortiz et al, 2004; Preciado, Pérez, Calzada y Preciado, 2005; Puyuelo y Llinás, 1992; Ortiz et al, 2004; Lemos y Rumel, 2005; Ossa, 2001; Escamilla, 1992; Escalona, 2007); sin embargo, en nuestra revisión bibliográfica no se encontraron estudios en los que se explicitaran mediciones ambientales de tiempo de reverberación e inteligibilidad en las aulas de clase.

Aunque, vale la pena destacar que Escalona (2007) y Gondim y Barreto (2004) en sus investigaciones realizaron mediciones ambientales de niveles de ruido de fondo, la primera tomó como indicador el Nivel Sonoro Equivalente Continuo (LEQ) y al final clasificó las escuelas en las que realizo el estudio en Ruidosas (LEQ > 70 dBA) y 
escuelas menos ruidosas (LEQ $<70 \mathrm{dBA}$ ); las segundas encontraron en las escuelas medidas índices de ruido insalubres.

Por su parte, Puyuelo y Llinas (1992) indagaron sobre condiciones acústicas cuestionando a los docentes evaluados acerca de la favorabilidad de las condiciones acústicas de las aulas y, si consideraban que los ruidos de fondo eran fuertes; sin embargo, no realizaron mediciones ambientales.

Preciado, Pérez, Calzada y Preciado (2005) tampoco realizaron mediciones ambientales argumentando que excedían el presupuesto asignado por sus patrocinadores, y tomaron la información referida por los docentes respecto a factores como la iluminación, polvo, ruido, acústica y eco; respecto a la acústica, el 49\% de los docentes reportó buena acústica, el 34\% regular, el 10\% bastante mala y el $7 \%$ mala.

En este orden de ideas, el hallazgo de condiciones acústicas muy poco favorables para el desarrollo de las actividades de construcción de conocimiento en las aulas evaluadas invita a indagarnos sobre las condiciones de trabajo de los docentes; en el caso de las condiciones físicas, los aspectos acústicos de un aula (tiempo de reverberación e inteligibilidad) se ven determinados no solo por las estructuras del espacio, sino también por la forma de desarrollo de la tarea, es asi, como por ejemplo, aspectos como el número de estudiantes puede ser determinante para la calidad acústica del aula, y por ende la inteligibilidad de la misma, lo cual de forma indirecta influye en la salud vocal y comunicativa de todos los actores del proceso pedagógico (docentes y estudiantes).

Por lo anterior, estamentos multisectoriales de la universidad deben proponer mecanismos que faciliten y garanticen el cubrimiento de la oferta educativa, respondiendo a las demandas que las políticas de orden nacional imponen a la universidad, pero sin perder de vista el mantenimiento de la calidad de la educación ofrecida por la misma, y de las condiciones de trabajo saludables de los docentes que laboran en ella y garantizan esta oferta educativa de calidad.

Como consideración final se sugiere la articulación activa de los diferentes estamentos universitarios en la planeación, diseño, implementación y articulación de las reformas físicas y curriculares de la universidad, con el objetivo de buscar el cumplimiento de las demandas que las políticas actuales exigen a las instituciones educativas (cubrimiento, rentabilidad, etc.) sin desmejorar o deteriorar las condiciones de salud-trabajo y vida de los docentes trabajadores de la institución, y por supuesto garantizando el mantenimiento de la calidad educativa que por décadas ha caracterizado a la universidad pública.

\section{CONCLUSIONES}

- Es recomendable que se realicen investigaciones posteriores que permitan analizar el impacto del número de estudiantes por aula sobre la acústica y el desgaste vocal de los docentes.

- Se sugiere la realización de estudios posteriores que incluyan mediciones acústicas y su impacto y relación con el número de estudiantes, las dimensiones y las estructuras de las aulas en la construcción de conocimientos en el aula, sin desconocer aquellos aspectos intraindividuales.

- Las instituciones educativas deber adaptar sus espacios para el desarrollo saludable de la labor docente, ya que las condiciones físicas (acústicas) inadecuadas afectan la salud de los participantes en el proceso de enseñanzaaprendizaje y las características de desarrollo de la tarea docente.

- A pesar del actual desarrollo tecnológico e informático se observa que las condiciones de realización de la tarea docente, y los procesos de construcción de conocimiento subyacentes no son todavía los óptimos para la salud y seguridad del docente como trabajador, y de los estudiantes como sujetos-objetos de trabajo. 
- La ausencia de estudios en los que se reporten mediciones objetivas de tiempo de reverberación e inteligibilidad demandó la necesidad de diseñar un índice de acústica total que diera razón de las condiciones acústicas de las aulas evaluadas, se sugiere que en el futuro se investigue más al respecto y se valide el mismo, ya que este se convierte en el primer estudio que analiza explícitamente estas variables, y estudios posteriores podrán aportar herramientas que permitan determinar por ejemplo, el numero optimo de estudiantes por aula sin desencadenar desgaste vocal en el docente; teniendo en cuenta la multicausalidad de los procesos de salud-enfermedad.

- Finalmente consideramos importante resaltar que el docente universitario está sumergido en una serie de dinámicas interaccionales y organizacionales que determinan las demandas y el desarrollo de la tarea docente; en la universidad participante aspectos como la carga académica, el número de estudiantes por clase, las condiciones físicos de las aulas de clase, la reforma académica actual, además de otras situaciones generan que las demandas a las que se debe enfrentar el profesor se incrementen, influyendo directamente en sus condiciones de salud-trabajo-vida.

\section{BIBLIOGRAFÍA}

1. Bureau International D'audiophonologie. BIAP. Recomendación biap 09/10-4. Inteligibilidad de la Palabra en las Aulas. (s/f). Recuperada el 22 de septiembre de 2008 en la web http://www.biap.org

2. Casas Sara, Klijn Tatiana. Promoción de la salud y un entorno laboral saludable. Revista Latino-am Enfermagem 2006. 14(1): 136-141.

3. Escalona Evelyn. Alteraciones de la voz y condiciones de trabajo en maestros de enseñanza Primaria. Aragua. Venezuela. La Habana. Cuba. Tesis presentada en opción al grado científico de Doctor en Ciencias de la Salud. Ministerio De Salud Pública. Escuela Nacional De Salud Pública; 2007.

4. Escamilla Indira. Frecuencia con la que se presentan problemas de voz en los profesores de la Universidad Nacional. Bogotá. Colombia. Tesis de grado; 1992.

5. Gondim M; Barreto M O estresse do professor acentuado pela precariedade das condições acústicas das salas de aula 2004. XXIV Encontro Nacional de Eng. de Produção. Florianópolis, SC, Brasil. 03 a 05 de nov.

6. Harris Cyril. Handbook of acoustical measurements and noise control. United States of America. McGraw Hill; 1991.

7. Lemos Simone, Rumel Davi. Ocorrência de disfonia em professores de escolas públicas da rede municipal de ensino de Criciúma-SC. São Paulo 2005. Revista Brasileira de Saúde Ocupacional, 30 (112): 07-13.

8. Miretti L, Santiago G. Modernidad y Políticas Educativas 2003. Informe de Avance presentado en el segundo Congreso Argentino de Administración Pública, Sociedad, Estado y Administración. Argentina.

9. Normas ISO 354 y UNE-EN-ISO 140-4. Medición del Tiempo de Reverberación.

10. Ortiz Erica, De Costa Everardo, Spina Ana, Crespo Agricio. Proposta de modelo de atendimento multidisciplinar para disfonias relacionadas ao trabalho: estudo preliminar 2004. Rev Bras Otorrinolaringol.V.70, n. 5, 590-596.

11. Ossa Luis. Salud y Calidad de Vida de los docentes de Risaralda. Investigación Colectiva desde la Pedagogía del Trabajo. Colombia. Editorial CÓDICE LTDA; 2001.

12. Preciado Julian, Pérez C, Calzada M, Preciado P. Frecuencia y factores de riesgo de los trastornos de la voz en el personal docente de La Rioja. Estudio transversal de 527 docentes: cuestionario, examen de la función vocal, análisis acústico y vídeolaringoestroscopia 2005. España. Acta Otorrinolaringológica Española. 55: 161-170.

13. Puyuelo Miguel y Llinas Maria. Problemas de voz en docentes 1992. Rev. Logop., Fon., Audiol., vol. 12. No. 2 (76-84).

14. Simões Marcia, Dias Maria. Prevalência de alteração vocal em educadoras e sua relação com a auto-percepção 2006. Rev Saúde Pública;40(6):1013-1018 


\section{ANEXO A. DETERMINACIÓN DE ÍNDICE DE ACÚSTICA TOTAL}

La acústica de un recinto está dada en función del Tiempo de Reverberación y la Inteligibilidad, sin embargo las mediciones ambientales realizadas en el presente estudio arrojaron información independiente de estos dos aspectos; por ello se optó por determinar un índice de Acústica Total analizando estas dos variables en cada salón.

Para la determinación del Índice de Acústica Total de los salones evaluados, a partir de los resultados obtenidos en las mediciones realizadas se diseñó la siguiente tabla:

\begin{tabular}{lll}
\hline Tiempo de Reverberación & Inteligibilidad & Acústica \\
\hline Bueno & Pobre & Regular \\
Bueno & Regular & Buena \\
Bueno & Bueno & Muy buena \\
Bueno & Excelente & Excelente \\
Malo & Pobre & Mala \\
Malo & Regular & Regular \\
Malo & Bueno & Buena \\
Malo & Excelente & Muy buena \\
Muy malo & Pobre & Muy mala \\
Muy malo & Regular & Mala \\
Muy malo & Bueno & Regular \\
Muy malo & Excelente & Buena \\
\hline
\end{tabular}

Fue a partir de la relación del Tiempo de Reverberación y la Inteligibilidad determinados en la Tabla del Índice de Acústica Total que se calificó cada salón evaluado. 